\title{
PREDICTION OF CRITICAL FLASHOVER VOLTAGE OF POLLUTED INSULATORS UNDER SEC AND RAIN CONDITIONS USING LEAST SQUARES SUPPORT VECTOR MACHINES (LS-SVM)
}

\author{
Abdelhalim MAHDJOUBI ${ }^{1}$, Boubakeur ZEGNINI ${ }^{1}$, Mohammed BELKHEIRI ${ }^{2}$ \\ ${ }^{1}$ Laboratory of studies and development of the Semiconducting and Dielectric Materials, LeDMaScD \\ Amar Telidji university of Laghouat, Laghouat (03000), Algeria \\ ${ }^{2}$ LTSS, Amar Telidji University of Laghouat, Laghouat (03000), Algeria \\ ah.mahdjoubi@lagh-univ.dz, b.zegnini@lagh-univ.dz, m.belkheiri@lagh-univ.dz
}

\begin{abstract}
This paper describes a methodology that was developed for the prediction of the critical flashover voltage of polluted insulators under sec and rain conditions least squares support vector machines (LS-SVM) optimization. The methodology uses as input variable characteristics of the insulator such as diameter, height, creepage distance, and the number of elements on a chain of insulators. The estimation of the flashover performance of polluted insulators is based on field experience and laboratory tests are invaluable as they significantly reduce the time and labour involved in insulator design and selection. The majority of the variables to be predicted are dependent upon several independent variables. The results from this work are useful to predict the contamination severity, critical flashover voltage as a function of contamination severity, arc length, and especially to predict the flashover voltage. The validity of the approach was examined by testing several insulators with different geometries. A comparison with the Grouping Multi-Duolateration Localization (GMDL) method proves the accuracy and goodness of LS-SVM. Moreover LS-SVMs give a good estimation of results which are validated by experimental tests.
\end{abstract}

Keywords: LS-SVM, flashover, modelling, polluted insulator, GMDL.

\section{INTRODUCTION}

Outdoor insulators can become heavily coated with dirt and chemicals by environmental pollution. In severe atmospheric conditions such as fog, dew or drizzle, the contaminant will were partially dissolved, forming a conductive layer. Leakage current flows along the surface will increase and may eventually cause flashover Insulators often undergo different kinds of pollution; among them, salt deposit near coasts, insect deposit or dust deposit. All this pollution can create weak areas on the surface of insulators which can cause a local discharge. Successively, those discharges can establish a flashover.

The critical flashover voltage of a polluted insulator is a significant parameter for the reliability of power systems. Several approaches have been developed for the estimation of the flashover voltage. Experimental tests are time-consuming and increase the cost of the system. To overcome such a problem researcher groups in high voltage engineering proposed some mathematical models based either on physical modeling, using electrical equivalent models or on mathematical regressions using artificial intelligent approximates [1-4]. The level and type of contamination of a region are associated with the pollution sources that produce it, as well as meteorological factors of the place.
When the weather influence is greater than pollution source influence, seasonal variations affect the tendency of contaminant accumulation over the insulator's surface. In these cases, the behavior of pollution level is very dynamic, coming to have in a same year highest and lowest pollution levels. Typical cases are the coasts [5]. A variety of prediction models have been proposed in the literature that include time-series models, regression models, artificial neural network (ANN) models, adaptive Neuro-fuzzy inference system (ANFIS) and support vector machine (SVM) models. The application of genetic algorithms enables the definition of the arc constants, resulting also in the calculation of the critical conditions at the beginning of the pollution flashover mechanism. A mathematical model has been established, which simulates accurately the experimental results [1, 5-7]. A new method like SVMs has been introduced for pattern recognition and regression [2]. SVMs have often been found to provide better prediction results [3]. As a simplification of traditional of SVM, Suykens et al. have proposed the use of the least squares support vector machines (LS-SVM) [4]. LS-SVM has been used for classification in various areas of pattern recognition [1], lately has been handled regression problems successfully [2]. In addition, experimental procedures introduced to treat flashover voltage of 
polluted insulator like X-ray fluorescence technique [8]; and Ultrasound technique based on ultrasonic noise emissions [9].

Another direction of research has been carried out recently based on least squares support vector machines LS-SVMs to estimate the critical flashover voltage and gaining from the global optimality of the achieved model

\section{BASICS OF LEAST SQUARE SUPPORT VECTOR MACHINES}

The formulation of LS-SVM is introduced as follows. $[1,5,6]$ Consider a given training set: $\left\{x_{i}\right.$, $\left.y_{i}\right\} \phi \in \mathfrak{R}^{2}, \mathrm{i}=1,2, \ldots \mathrm{N}$ With input data $x_{i}$, and output data $y_{i}$

The following regression model can be constructed by using non-linear mapping function $\phi$

$$
y=w^{T} \phi(x)+b
$$

Where $\mathrm{w}$ is the weight vector and $\mathrm{b}$ is the bias term. As in SVM, it is necessary to minimize a cost function $\mathrm{C}$ containing a penalized regression error, as follows:

$$
\min C(w, e)=\frac{1}{2} w^{T} w+\frac{1}{2} \gamma \sum_{i=1}^{N} e_{i}^{2}
$$

Subject to equality constraints

$$
y=w^{T} \phi\left(x_{i}\right)+b+e_{i}, \mathrm{i}=1,2 \ldots, \mathrm{N}
$$

The first part of this cost function is a weight decay which is used to regularize weight sizes and penalize large weights. Due to this regularization, the weights converge to similar value. Large weights deteriorate the generalization ability of the LS-SVM because they can cause excessive variance. The second part of (2) is the regression error for all training data. The parameter $\mathrm{C}$, which has to be optimized by the user, gives the relative weight of this part as compared to the first part. The restriction supplied by (3) gives the definition of the regression error. To solve this optimization problem, Lagrange function is constructed as mentioned in (4). $\alpha_{i}$ are Lagrange multipliers.

$$
\begin{aligned}
L(w, b, e, \alpha) & =\frac{1}{2}\|w\|^{2}+\gamma \sum_{i=1}^{N} e_{i}^{2} \\
& -\sum_{i=1} \alpha_{i}\left\{w^{T} \phi\left(x_{i}\right)+b+e_{i}-y_{i}\right\}
\end{aligned}
$$

The solution of (4) can be obtained by partially differentiating with respect to $w, b, e_{i}$ and $\alpha_{i}$ which gives:

$$
w=\sum_{i=1}^{N} \alpha_{i} \phi\left(x_{i}\right)=\sum_{i=1}^{N} \gamma e_{i} \phi\left(x_{i}\right)
$$

Where a positive definite Kernel is used as follows:

$$
K\left(x_{i}, x_{j}\right)=\phi\left(x_{i}\right)^{T} \phi\left(x_{j}\right)
$$

An important result of this approach is that the weights $(w)$ can be written as linear combinations of the Lagrange multipliers with the corresponding data training $\left(x_{i}\right)$. Putting the result of (5) into (1), the following result is obtained:

$$
y=\sum_{i=1}^{N} \alpha_{i} \phi\left(x_{i}\right)^{T} \phi(x)+b
$$

For a point $y_{i}$ to be evaluated it is:

$$
y_{i}=\sum_{i=1}^{N} \alpha_{i} \phi\left(x_{i}\right)^{T} \phi\left(x_{j}\right)+b
$$

Depending on the number of training data set either direct solvers can be used or an iterative solver such as conjugate gradients methods (for large data sets), in both cases with numerically reliable methods. In applications involving nonlinear regression it is enough to change the inner product of $\left\langle\Phi\left(x_{i}\right), \Phi\left(y_{i}\right)\right\rangle$ (7) by a kernel function and the $\mathrm{ij}^{\text {th }}$ element of matrix $\mathrm{K}$ equals to (5)

This leads to the following nonlinear regression function:

$$
y=\sum_{i=1}^{N} \alpha_{i} K\left(x_{i}, x\right)+b
$$

For a point $x_{j}$ to be evaluated it is:

$$
y_{j}=\sum_{i}^{N} \alpha_{i} K\left(x_{i}, x_{j}\right)+b
$$

For LS-SVM, there are many kernel functions (linear, polynomial, radial basis function (RBF), spline, bspline, sigmoid, etc. However, the more used kernel function is RBF, a simple Gaussian function. It is defined by (11). $\sigma_{s v}{ }^{2}$ is the squared variance of the Gaussian function. It should be optimized by the user, to obtain the support vector. $\alpha$ of the RBF kernel should be stressed and it is very important to make a careful model selection of the tuning parameters, in combination with the regularization constant $\gamma$, in order to achieve a good generalized model.

$$
K\left(x_{i}, x_{j}\right)=\exp \left(-\frac{\left\|x_{i}-x_{j}\right\|^{2}}{\sigma_{s v}{ }^{2}}\right)
$$

\section{COMPUTATION OF CRITICAL FLASHOVER VOLTAGE OF POLLUTED INSULATORS USING LS-SVM}

LS-SVM is the variant that modifies the original SVM formulation, leading to solving a set of linear equations that is easier to solve than problems, while most of the important advantages of SVM are retained. The electrical flashover voltage can be written like mentioned in (12) [1]. $R_{p}$ denotes the uniform surface resistance per unit length of the pollution layer, $N$ is the reigniting constant, $a$ is the arc equation exponent

$$
E_{c}=N^{(1 /(a+1))} \cdot R_{p}^{(a /(a+1))}
$$

To measure the ESDD (equivalent salt deposit density) of polluted insulators, we use this criterion (13) [10]. $C$ is the salinity $\left(\mathrm{mg} / \mathrm{m}^{3}\right), Q$ denotes the distilled water $\left(\mathrm{cm}^{3}\right)$ and $A$ present the area of the insulator surface. 


$$
E S D D=\frac{C \cdot Q}{A}
$$

LS-SVM evaluates the given patterns and it can be produce new outputs for different inputs. It must be trained well by help of obtained dependable patterns. In this study a training set containing adequate representative data points is constructed. This is a considerable step in building an LS-SVM model that can represent insulators critical flashover voltage. In this work, insulator height $(H$ $m m)$, insulator diameter $\left(D_{m}-m m\right)$, leakage length of the insulator of an element $(L-m m)$, and number of elements in a chain of insulators are used as input vector to the approximate and the flashover critical voltage $(V c-k V)$ as the output of the approximate in two cases; sec condition and rain condition. Therefore, the training set with input-output data was constructed in the training process. We used in this study twelve kinds of insulators; $U 70 B S$, U160BS, U300B, U400B, U530B, U160BSP, $U 300 B P, U 120 A D, U 160 A D, U 210 A D, 52-3$ and 52-8 [11]. The characteristics of these insulators are given in Table I. Each pattern of the training set contains six inputs which characterize parameters of $H, D_{m}, L, N E$, and one output which represent $V_{C}$ critical flashover voltage. Constructing a testing set is also a significant step in evaluating the prediction performance of the LS-SVM model with representative data points of different insulators that are not used during training. We used for each kind of insulators 22 training data and 7 test data.

In global, we have 264 training data and 84 testing data.

Table 1. Characteristics of the investigated insulators [11].

\begin{tabular}{|l|l|l|l|}
\hline Insulator type & $D_{m}(\mathrm{~mm})$ & $H(\mathrm{~mm})$ & $L(\mathrm{~mm})$ \\
\hline$U 70 B S$ & 255 & 127 & 320 \\
\hline$U 160 B S$ & 285 & 146 & 325 \\
\hline$U 300 B$ & 320 & 195 & 390 \\
\hline$U 400 B$ & 360 & 205 & 555 \\
\hline$U 530 B$ & 360 & 240 & 600 \\
\hline$U 300 B P$ & 360 & 146 & 545 \\
\hline$U 300 B P$ & 360 & 195 & 617 \\
\hline$U 120 A D$ & 380 & 127 & 365 \\
\hline$U 160 A D$ & 420 & 146 & 400 \\
\hline$U 210 A D$ & 420 & 170 & 400 \\
\hline $52-3$ & 255 & 146 & 320 \\
\hline $52-8$ & 280 & 146 & 385 \\
\hline
\end{tabular}

The computation is divided into two sections; the first is to find the critical flashover voltage in sec conditions and the second in rain condition; the data of experimental tests are used from previous researches [11]. The search is tuned to a finer search in the region where the predicted RMSE take minimum values with the optima LS-SVM parameters (Fig. 1). To achieve a high level of performance with LS-SVM model, some parameters have to be tuned, including the regularization parameter $\gamma$ and the kernel parameter $\sigma$.



Fig. 1. RMSEV in (a) sec (b) rain conditions

The optima values are obtained by manually searc of parameters which corresponding to:

- $\sigma^{2}=11.3137$ and $\gamma=6.7110^{7}$ in sec case;

$-\sigma^{2}=4$ and $\gamma=6.7110^{7}$ in rain case.

The results of the model proposed with LSSVM method is calculated and give best results which are shown later in figures 3 and 4. Many papers have used different artificial neural network topologies (Radial basis functions and multilayer perceptron) for the estimation of the flashover voltage on insulators $[12,13,14]$ but the construction of the neural network, fixing and the number of neurons per layer, and the generalization ability presents always a serious problem in this direction. LS-SVM strategy using a fixed set of support vectors is proposed to evaluate the flashover performance of outdoor insulators under in different weather conditions.

LS-SVM gives more efficiency of results according to past researches made like presented by M. T. Gençoğlu, M. Uyar [1]. They predicted results using RBF kernel with an accuracy of $99.97 \%$, whereas with MFNN (Feed forward Neural Networks) gives an accuracy of $99.89 \%$; for that LSSVM is more powerful in the prediction of flashover voltage on insulators.

Based on results obtained, we can note that the flashover voltage in rain condition is lower than shown in sec condition. That explains the effect change of resistance $R_{p}$ by the deposit of water drops on the surface of insulators. The optimization with LS-SVM done best results like shown previously. Data used are tested by Global insulator 
group with conformity with GOST 6490-93 and CEI 60383 [15].

The results are testing by the accuracy:

- $R^{2}=99.70 \%$ in sec condition;

- $R^{2}=99.83 \%$ in rain condition.

In this context, we add an another test made by using Grouping Multi Duolateration Localization (GMDL) method based on artificial neurons networks like made by Hojae Lee et al. [15]. V.T. Kontargyri et al. [16]. are used this approach of ANN to evaluate the flashover voltage on insulators. After they thought to improve the ANN algorithm to get best results like made by Hadi Fattahi and Habibollah Bazdar to evaluate drilling rate index [17]. Armando Souza Guedesa et al. [18] are using this approach to evaluate the electrical insulation in three-phase induction motors. GMDL give amelioration to the ANN classic by the elimination of bad layers and improving the best layers of the network. This method is cleared on the flow chart (Figure 2).

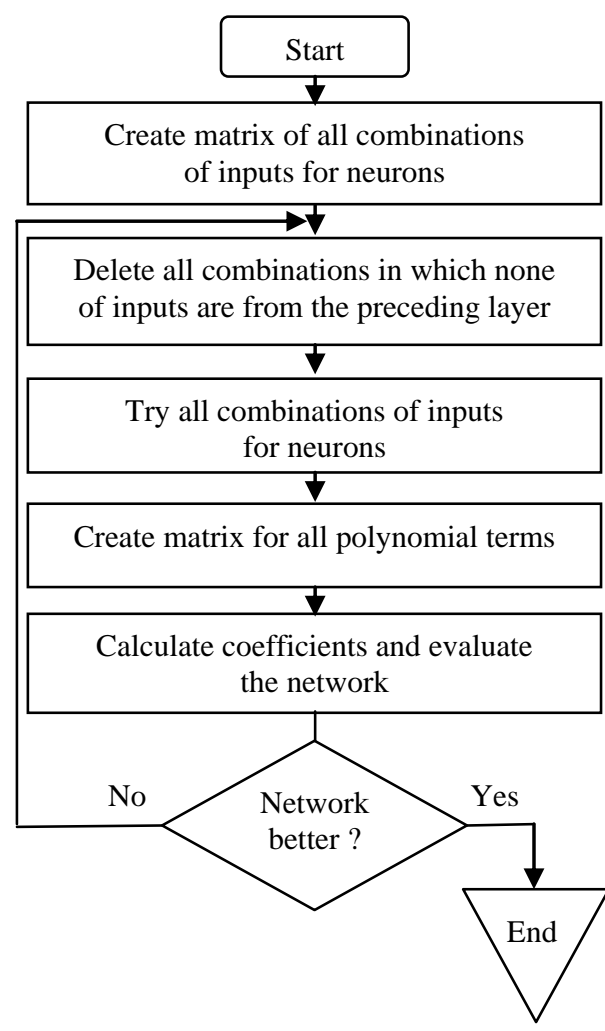

Fig. 2. GMDL flow chart

LS-SVM proves a good robustness and better results. The accuracy of GMDL method was:

- $\quad R^{2}=99.29 \%$ in sec condition;

- $\quad R^{2}=96.89 \%$ in rain condition.

Compared to other intelligent techniques in modeling fashover, we see clearly that the proposed model achieves higher correlation coefficient $\mathrm{R}=0.9987$ with very small root mean square error (RMSE=0.0389) and outperforms other modeling strategies.
Table 2: Comparison of the proposed LS-SVM models with others modeling strategies.

\begin{tabular}{|l|l|l|l|}
\hline $\begin{array}{l}\text { modelling } \\
\text { strategies }\end{array}$ & $\begin{array}{l}\text { root mean } \\
\text { square } \\
\text { error } \\
\text { (RMSE), }\end{array}$ & $\begin{array}{l}\text { mean } \\
\text { absolute } \\
\text { error } \\
\text { (MAE \%) }\end{array}$ & $\begin{array}{l}\text { coefficient of } \\
\text { determination } \\
\left(\mathrm{R}^{2}\right)\end{array}$ \\
\hline ANN [19] & & 3.8400 & 0.9853 \\
\hline $\begin{array}{l}\text { Fuzzy } \\
\text { Logic [16] }\end{array}$ & & & 0.9670 \\
\hline ANFIS [20] & 0.0473 & 0.4597 & 0.9910 \\
\hline GMDL sec & & & 0.9929 \\
\hline GMDL rain & & & 0.9989 \\
\hline $\begin{array}{l}\text { LS-SVM } \\
\text { sec }\end{array}$ & 0.0389 & & 0.9970 \\
\hline $\begin{array}{l}\text { LS-SVM } \\
\text { rain }\end{array}$ & 0.0371 & & 0.9983 \\
\hline
\end{tabular}

The results of comparison are shown in figures 3 and 4.

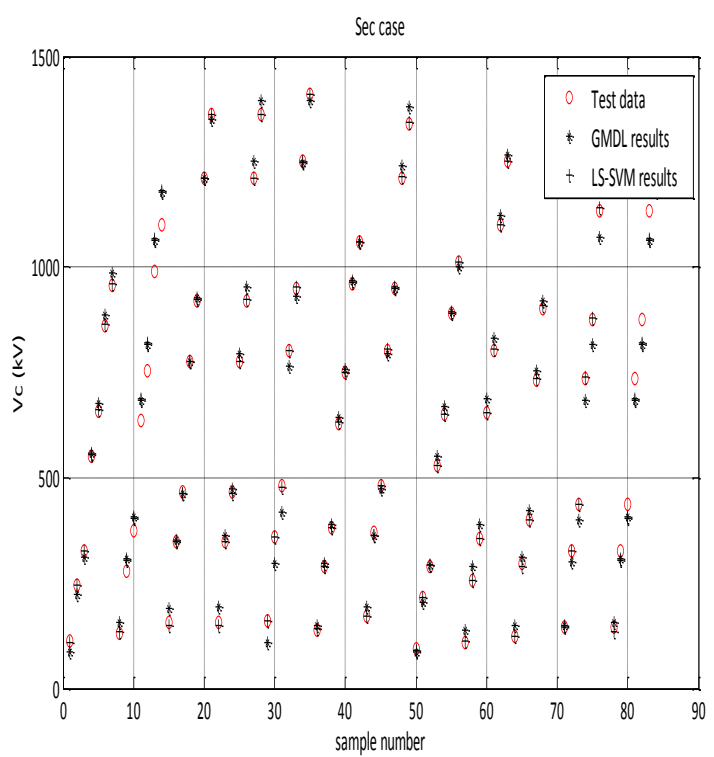

Fig. 3. Comparison flashover voltage in sec conditions

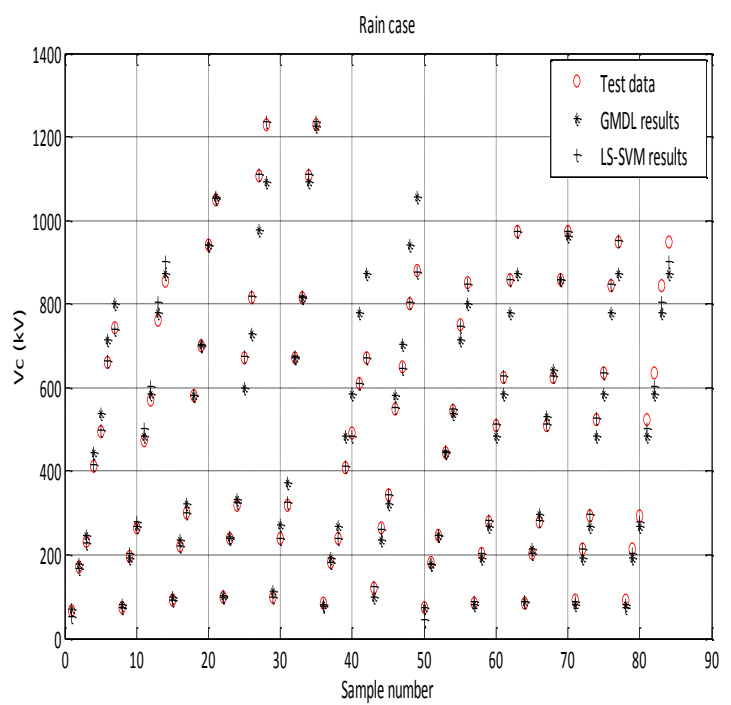

Fig. 4. Comparison flashover voltage in rain conditions 
By comparison between values of flashover voltage in sec condition and rainy, we can do the mean proportion like:

$$
\frac{V_{\mathrm{sec}}}{V_{\text {rain }}} \approx 1.362
$$

In our study, the LS-SVM approach is based on radial basis function (RBF); we can use other kernel functions like simple Gaussian function, polynomial function and multi-layer perceptron for more results and make comparison. The results obtained are satisfactory, but for more goodness we can expand data size. The most common approach is used at many sites of leakage current monitoring. Slama, M. El.-A and al have done a systematic study of the leakage current of high voltage polyamide insulator string under dry and wet tests [21], the leakage current increase linearly with the applied voltage. This result indicates that there is no apparition of dry arcs at the insulator surface, traces of erosion and tracking of insulators resulting of partial discharges are observed. Now we prepare experimental test in our laboratory to measure leakage current by inserting a precision unity value resistor or special purpose current transducer(e.g. a CT with high permeability core) in series with insulator and to measure voltage across it. This voltage is then translated to actual values by some microcontroller and/or computer system and software. By using the combinations of sensors, processing techniques \& electronics set up, the characteristics of the leakage current can be evaluated. For more results and especially gives an overview on the return stroke currents along the chain of insulators. The presented technique can help high voltage engineers to assess outdoor insulator performance under in different weather conditions.

\section{CONCLUSION}

In this paper, the LS-SVM model are developed in order to determine the relationship between critical flashover voltage as a function of insulator height, insulator diameter, leakage length of the insulator for an element, the number of elements on a chain. Two kinds of conditions are used in this study; sec condition and rainy in order to show the influence of water drops of rain in decreasing of flashover voltage. The comparisons and the results presented in this investigation, such as with other methods like GMDL model, reveal clearly the capability of the proposed LS-SVM modeling strategy in predicting critical flashover voltage values of different insulator types in any region by giving detailed information of electrical transmission system.

\section{REFERENCES}

1. Gencoglu MT, Uyar M. Prediction of flashover voltage of insulators using least squares support vector machines. Expert Systems with Applications. 2009; 36: 10789-10798.

2. Sundararajan R, Gorur RS. Effect of insulator profiles on dc flashover voltage under polluted conditions: A study using a dynamic arc model. IEEE Transaction on Dielectrics and Electrical Insulation. 1994; 1(1):124-132. https://doi.org/10.1109/94.300239

3. Rizk FAM. A criterion for AC flashover of contaminated insulators. In IEEE, (PESWPM) Power Engineering Society Winter Power Meeting, New York, NY, USA. 1971;90: 135

4. Rizk FAM. Mathematical models for pollution flashover. ELECTRA. 1981; 78: 71-103.

5. Gerardo Montoya, Isa'ias Ram'irez, Jorge I. Montoya. Measuring pollution level generated on electrical insulators after a strong storm. Electric Power Systems Research. 2004; 71: 267-273.

6. Zegnini B, Mahdjoubi AH, Belkheiri M. A least squares support vectors machines (LS-SVM) approach for predicting critical flashover voltage of polluted insulators. Annual report conference on electrical insulation and dielectric phenomena (CEIDP). 2011: 403-406. https://doi.org/10.1109/CEIDP.2011.6232680

7. Belkheiri M, Zegnini B, Mahi D. Modeling of the critical flashover voltage of high voltage insulators using artificial intelligence, (JICA) Journal of intelligent Computing and Applications, Serial publications. 2009; 2(2): 137-154.

8. Shigemasa Enomoto, Kuniaki Nishioka, Noshio Higashiyama, Atsushi Sasaki. Measurement of Salt Surface Density on Polluted Insulators Using a Simple x-Ray Fluorescence Technique, ELSEVIER (IJRAI) International Journal of Radiation Applications and Instrumentation. 1992; 43(5): 615620.

9. Tarso V, Ferreira, André D, Germano, Edson Guedes da Costa. Ultrasound and Artificial Intelligence Applied to the Pollution Estimation in Insulations. IEEE transactions on power delivery. 2012; 27(2): 583-589. https://doi.org/10.1109/TPWRD.2011.2178042

10. Osama E, Gouda1, Adel Z. El Dein. Simulation of overhead transmission line insulators under desert environments, (IET-GTD) Generation, Transmission and Distribution. 2013; 7(1): 9-13. https://doi.org/10.1049/iet-gtd.2011.0778

11. Global Insulator Group, Isolateurs pour lignes de transmission et stations de distribution à tension de 0,4 à $1150 \mathrm{KV}$, catalogue des produits, Ukraine. 2012.

12. Gencoglu MT, Cebeci M. The pollution flashover on high voltage insulators. Electric Power Systems Research. 2008; 78(11): 1914-1921. https://doi.org/10.1016/j.epsr.2008.03.019

13. Ahmad AS, Ghosh P, Ahmed S, Aljunid SAK. Assessment of esdd on high-voltage insulators using artificial neural network. Electric Power Systems Research. 2004; 72(2):131-136. 
14. Zegnini B, Belkheiri M, Mahi D. Modeling fashover voltage (fov) of polluted hv insulators using artificial neural networks (anns) 2009 International Conference on Electrical and Electronics Engineering ELECO. 2009: 336-340. https://doi.org/10.1109/ELECO.2009.5355301

15. Hojae Lee, Sanghoon Lee; Yeonsoo Kim, Hakjin Chong. Grouping multi-duolateration localization using partial space information for indoor wireless sensor networks. IEEE Transactions on Consumer Electronics. 2009;55(4):1950-1958.

16. Kontargyri VT, Gialketsi AA, Tsekouras GJ, Gonos IF, Stathopulos IA. Design of an artificial neural network for the estimation of the flashover voltage on insulators. (EPSR) Electric Power Systems Research. 2007; 77:1532-1540.

17. Hadi Fattahi, Habibollah Bazdar, Applying improved artificial neural network models to evaluate drilling rate Index. Tunnelling and Underground Space Technology. (2017);70: 114124.

18. Armando Souza Guedesa, Sidelmo Magalhães Silvaa, Braz de Jesus Cardoso Filhoa, Cláudio Alvares Conceic. Evaluation of electrical insulation in three-phase induction motorsand classification of failures using neural networks. Electric Power Systems Research. 2016; 140:263-273. https://doi.org/10.1016/j.epsr.2016.06.016

19. Asimakopoulou GE; Kontargyri VT, Tsekouras GJ, Elias CN, Asimakopoulou FE, Stathopulos IA. A fuzzy logic optimisation methodology for the estimation of the critical flashover voltage on insulators. Electric Power Systems Research. 2001; 81(2):580-588.

https://doi.org/10.1016/j.epsr.2010.10.024.

20. Erenturk K. Adaptive-network-based fuzzy inference system application to estimate the flashover voltage on insulator. Instrumentation Science \& Technology. 2009; 37(4):446-461.

21. Slama M, Abderrahmane Beroual El-A. Behavior of AC High Voltage Polyamide Insulators: Evolution of Leakage Current in Different Surface Conditions. Power Engineering and Electrical Engineering. 2015; 13(2): 74-80 https://doi.org/10.15598/aeee.v13i2.1145.

Received 2018-06-28

Accepted 2018-11-19

Available online 2018-11-27

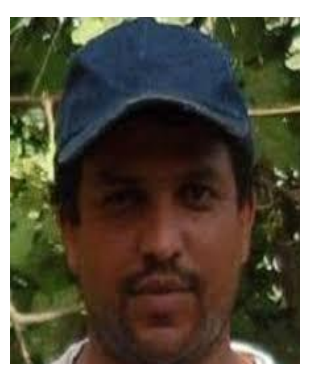

Abdelhalim MAHDJOUBI

was born in 1975 in Algeria. He obtained his engineer degree in electrical engineering from the University center of Laghouat in June 1998, and the magister degree in Dielectrics Materials from the institute of Electrical Engineering, University Center of Laghouat (Algeria) in 2002. $\mathrm{He}$ received the $\mathrm{PhD}$ degree in Electrical engineering from University of Amar Telidji Laghouat ( Algeria) in September 2014. His research interests concern :Insulation materials, High voltage, Modeling, Simulation, Artificial intelligence.

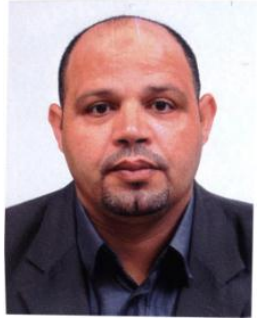

Boubakeur ZEGNINI was born in Laghouat, Algeria on January 25, 1968. He received the Dipl. Eng. Degree in electrical engineering from the ENSET of Laghouat (Algeria) in 1991, the M.Sc. degree from the institute of Electrical Engineering, University Center of Laghouat (Algeria) in 2001. He received the $\mathrm{PhD}$ degree in electrical engineering from Mohammed Boudiaf Science and Technology University of Oran - USTMB Oran (Algeria) in 2007.

$\mathrm{He}$ is full Professor at the University of Amar Telidji, in Laghouat (Algeria). His research interests concern: Material characterization, Insulation materials, High Voltage, Modelling, Simulation, Artificial Intelligence, and Power Systems. He supervised more than 12 doctors of Electrical Engineering, dozens of graduates of Magister's and Materials for Electrical Engineering studies. He is author / Co-author of more than 95 technical papers including more than 30 refereed journal papers, international conference, and 1 book chapter.

Correspondence address: Laboratory of studies and Development of Semiconductor and Dielectric Materials, LeDMaScD, University Amar Telidji of Laghouat, BP $37 \mathrm{G}$ route of Ghardaïa, Laghouat 03000, Algeria.

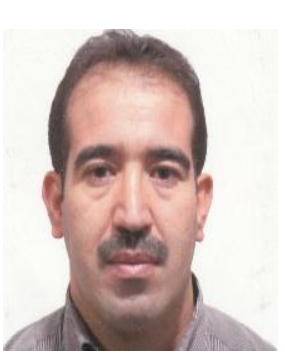

Mohammed BELKHEIRI was born in 1977 in Algeria. He obtained his engineer degree in electrical engineering from the University of Boumerdès in June 2000 , and the magister degree in Robotics and Automatic Control from Military Polytechnic School of Algiers in Nov 2002. He received the $\mathrm{PhD}$ degree in automatic control from Ecole Nationale Polytechnique d'Alger 2008.

He worked as an assistant professor from 2003 to 2008 then as an associate professor in University of Laghouat from 2008 to 2016 . He is currently working as a professor in electrical engineering department at university of Laghouat. $\mathrm{He}$ joined the Telecommunications, signals and systems research laboratory since 2011. His research interests include nonlinear, adaptive and intelligent Neural Networks control of electromechanical systems and robotics. 